\title{
Heterogeneous Reasoning in Real Arithmetic
}

\author{
M. Urbas and M. Jamnik \\ University of Cambridge \\ \{Matej.Urbas, Mateja.Jamnik\}@cl.cam.ac.uk
}

\begin{abstract}
Diagrams often complement sentential proofs in mathematics. However, diagrams are rarely used as standalone reasoning tools. Thus we propose to integrate diagrammatic reasoning with an existing sentential theorem prover, thus enabling so-called heterogeneous reasoning, particularly in real arithmetic. We will study a set of diagrammatic proof examples from which we will construct a diagrammatic language, inference rules and communication procedures between the diagrammatic and sentential reasoners. The resulting framework will allow the use of diagrammatic proof steps in the same way as the sentential ones, all within the same attempt to construct a proof.
\end{abstract}

\section{Introduction}

Most diagrammatic reasoning approaches are strictly informal (e.g., sketches or specific illustrations of a general problem). This lead to numerous diagrammatic formalisation efforts $[2,3,5]$. However, proofs "on paper" rarely consist exclusively of drawings. Diagrams are often accompanied by sentential formulae. This motivated some to investigate heterogeneous reasoning $[1,8]$.

Our goal is to introduce diagrammatic reasoning techniques into an existing sentential theorem prover, thus devising a heterogeneous reasoner. We first study heterogeneous proof examples ${ }^{1}$ in real arithmetic, from which we will then construct diagrammatic inference rules and language. Finally we will integrate the two modes of reasoning - the diagrammatic logic into the sentential prover.

One of our goals is to show whether heterogeneous reasoning can improve proof intuitiveness in sentential provers. We also believe that heterogeneous methods can provide better or entirely novel proof hints. Hints in homogeneous sentential systems are provided in residual statements of an unsuccessful proof attempt. The unresolved statements can be inspected for clues on how to proceed [4]. However, such hints are often not easily discernible even for experts.

Additionally, naive general inferences from specific diagrams can result in incorrect conclusions. ${ }^{2}$ Thus it is essential to provide a suitable diagrammatic formalism. Our aims can be broken down into several sub-goals:

Diagrammatic formalisation: Introduce diagrammatic inference rules for our logic, check soundness and ensure that the language is powerful enough to cover a sufficiently large subset of problems in the target domain.

Diagrammatic reasoner: Construct a diagrammatic reasoner that can either prove a goal or produce a transformed one, which can again be used in the sentential reasoner or act as a hint.

\footnotetext{
${ }^{1}$ Examples were taken from Nelsen's Proofs without words [7].

${ }^{2}$ A famous example is Cauchy's erroneous proof of the Euler characteristic for all polyhedra [6] This "proof" remained unchallenged for decades.
} 
Integration: We have to establish a bidirectional translation between the two modes of reasoning and integrate the diagrammatic reasoner into the chosen sentential theorem prover. The truth of all statements must be preserved during the translation. Also, integration must allow not only diagrammatic proof steps, but also conversion of theorems and statements between the two realms.

\section{Heterogeneous Reasoning}

We currently identify three types of heterogeneous interactions (non-exhaustive, based on our proof examples):

1.) Statement transform: Diagrammatic transformations are used to rewrite a sentential statement into some other equivalent statement (Fig. 1).

2.) Introduction of new goals: $\mathrm{Di}-$ agrammatic transformations introduce a set of new statements or lemmas (Fig. 2).

3.) Proving lemmas: Entire lemmas can be proved with diagrammatic methods. An example is the proof of $\lim _{n \rightarrow \infty} \sum_{i=1}^{n} \frac{1}{2^{i}}=1$. Fig. 3 illustrates a possible lemma for this theorem, which is proved diagrammatically. Afterwards we use the sentential reasoner to prove $\lim _{n \rightarrow \infty} \frac{1}{2^{n}}=0$. This last rule is then used to eliminate $\mathrm{B}=\frac{1}{2^{n}}$ in the diagrammatic proof.

Our target domain is the field of real

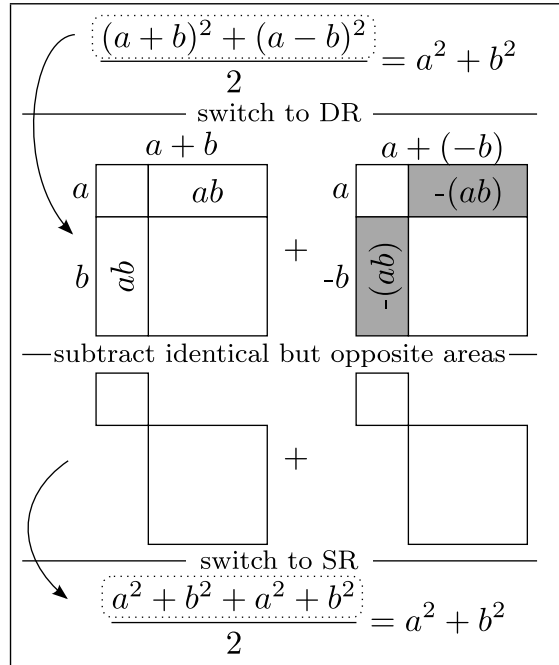

Fig. 1. Diagrammatic statement rewrite. Gray denotes the opposite sign of the area - same size areas of opposite sign cancel each-other when combined.

arithmetic, that is formulae from the ordered field $[\mathbb{R},+, \cdot, 0,1,<]$. Numbers and variables are represented as edges and rectangular areas. Areas also act as multiplication of edges. Summation is represented by multiple areas and connected edges that extend in the same direction. Also, we use gray to denote the sign of objects. Universal quantification is implicit in the diagram for all variables.

\section{Methodology}

In order to devise a framework with which we can construct heterogeneous proofs as the ones described above, we need to complete the following tasks:

- Define a precise description of the diagrammatic language and its formal inference rules. We will study several examples to determine the required features of the language and the set of inference rules.

- Secondly, we will study the reasoning and theory formalisms in the sentential reasoner. With this, we will determine how the logic of the reasoner influences our diagrammatic language. Because of local expertise, we chose Isabelle [9] as the underlying interactive prover. Initially, the user will choose when to switch between the two modes. Later, we will examine the automation of this choice. 


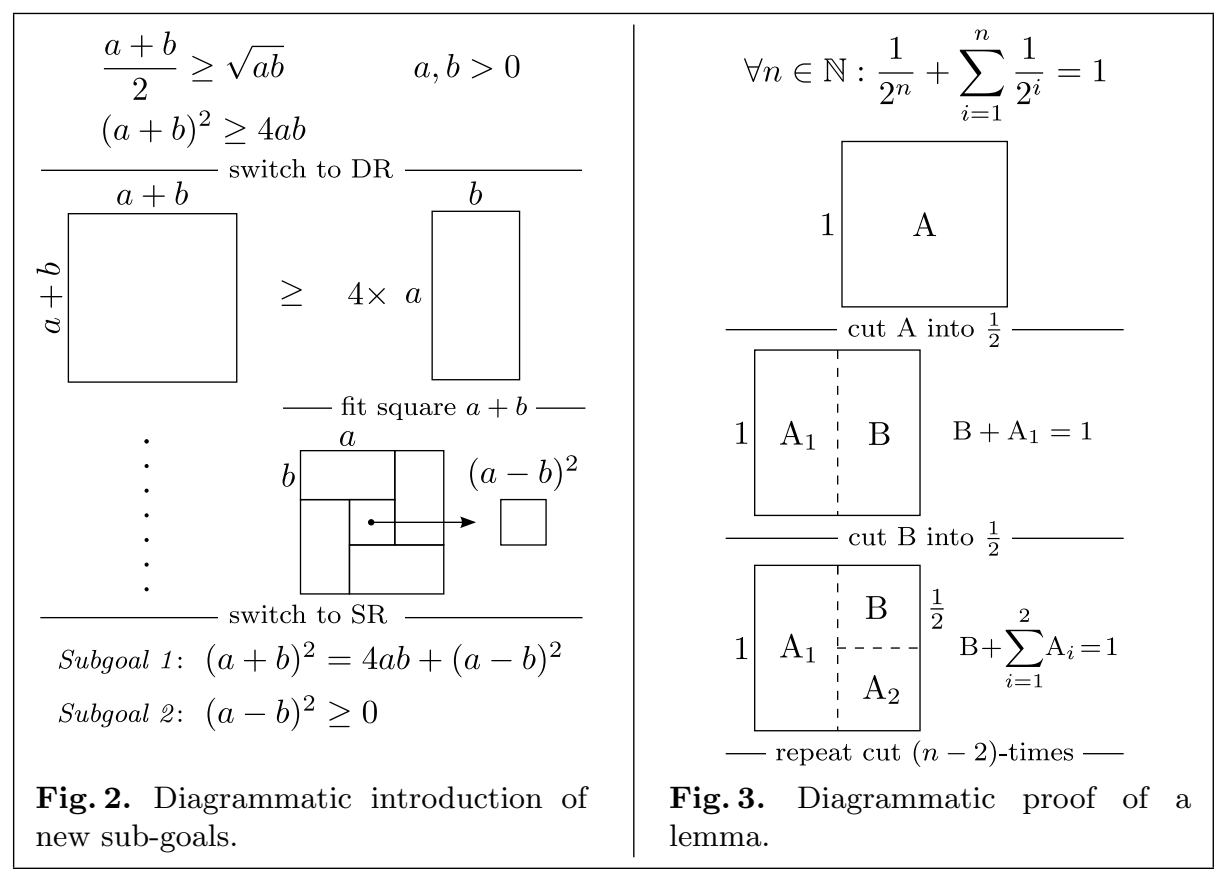

- In the last phase, we will design a communication link between the diagrammatic and symbolic representations. We have chosen a heterogeneous framework architecture where the diagrammatic tactics and statements represent extensions to the built-in native symbolic set of instructions in Isabelle. This will require translation or reuse of internal structures of Isabelle.

In summary, there are many ways in which heterogeneous reasoning can complement sentential approaches, e.g.: more intuitive proofs and proof hints, novel proof tactics, and greater expressive power. We believe that extending a sentential theorem prover with diagrammatic reasoning is viable and advantageous.

\section{References}

1. J. Barwise and J. Etchemendy. A Computational Architecture for Heterogeneous Reasoning. In TARK, pages 1-11. Morgan Kaufmann, 1998.

2. E. Hammer. Reasoning with Sentences and Diagrams. NDJFL, 35(1):73-87, 1994.

3. J. Howse and G. Stapleton. Visual Mathematics: Diagrammatic Formalization and Proof. LNCS, 5144:478-493, 2008.

4. A. Ireland, M. Jackson, and G. Reid. Interactive Proof Critics. JFAC, 11(3):302$325,1999$.

5. M. Jamnik, A. Bundy, and I. Green. On Automating Diagrammatic Proofs of Arithmetic Arguments. JOLLI, 8(3):297-321, 1999.

6. I. Lakatos. Proofs and Refutations: The Logic of Mathematical Discovery, 1976.

7. R. B. Nelsen. Proofs without Words: Exercises in Visual Thinking, 1997.

8. S.-J. Shin. Heterogeneous Reasoning and its Logic. BSL, 10(1):86-106, 2004.

9. M. Wenzel, L. C. Paulson, and T. Nipkow. The Isabelle Framework. In TPHOLs, pages 33-38, 2008. 